\title{
BMJ Open Clinical practitioners' views on the management of patients with medically unexplained physical symptoms (MUPS): a qualitative study
}

\author{
A Keith W Brownell, ${ }^{1}$ Chloe Atkins, ${ }^{2}$ Andrea Whiteley, ${ }^{3}$ Robert F Woollard, ${ }^{4}$ \\ Jude Kornelsen ${ }^{4}$
}

To cite: Brownell AKW, Atkins C, Whiteley A, et al. Clinical practitioners' views on the management of patients with medically unexplained physical symptoms (MUPS): a qualitative study. BMJ Open 2016;6: 012379 .

doi:10.1136/bmjopen-2016012379

- Prepublication history for this paper is available online. To view these files please visit the journal online (http://dx.doi.org/10.1136/ bmjopen-2016-012379).

Received 26 April 2016 Revised 24 August 2016 Accepted 28 October 2016

CrossMark

For numbered affiliations see end of article.

Correspondence to Dr A Keith W Brownell; akwb@shaw.ca

\section{ABSTRACT}

Objectives: By identifying strategies that practicing physicians use in managing patients with medically unexplained physical symptoms (MUPS), we present an interim practical management guide (IPMG) that clinical practitioners may find useful in their clinical practices and that may help guide future research.

Design: A qualitative research study based on interview data from practicing physicians with experience in dealing with MUPS and known to the physician members of the research team. A parallel exploration of patient experiences was carried out simultaneously and is reported elsewhere.

Setting: 2 urban centres in 2 different Canadian provinces in a healthcare system where family physicians provide the majority of primary care and self-referral to specialists rarely occurs.

Participants: The physician members of the research team invited practicing family and specialty physicians to participate in the study.

Results: We characterise the care of patients with MUPS in terms of a 4-part framework: (1) the challenge of diagnosis; (2) the challenge of management/treatment; (3) the importance of communication and (4) the importance of the therapeutic relationship.

Conclusions: On the basis of the details in the different parts of the framework, we propose an IPMG that practitioners may find useful to facilitate the clinical care of patients with MUPS. The guide can be readily implemented into the practice of any physician who cares for patients with MUPS.

\section{INTRODUCTION}

In this era of burgeoning technology, remarkable scientific breakthroughs and evidencebased medical practice, there is a cultural expectation that physicians will be able to diagnose and treat illnesses. Yet, primary care and specialist physicians frequently encounter patients with medically unexplained physical symptoms (MUPS). ${ }^{1-3}$ When the symptoms

\section{Strengths and limitations of this study}

- The multidisciplinarity of the research team broadened the perspectives brought to the analysis of the data.

- The interim practical management guide (IPMG) recommendations are based on data obtained from primary care and specialist physicians who care for patients with medically unexplained physical symptoms (MUPS) in two different Canadian provinces.

- The recommendations for practice provide an IPMG for the management of the patient with MUPS.

- Future validation and potential refinement of the interim guide in a large group of practitioners need to be performed.

- As this study was performed in Canada, a wealthy country with a universal governmentfunded healthcare system, where family physicians provide the majority of primary care, the generalisability of the recommendations for other healthcare delivery systems is unknown.

are persistent and a traditional medical diagnosis and treatment is not forthcoming, the terms MUPS or medically unexplained symptoms (MUS) may be used by some practitioners as a diagnostic placeholder. ${ }^{45}$

In a 2013 article, Aiarzaguena et al succinctly summarise current challenges for physicians dealing with patients with MUPS:

Relations between physicians and patients with medically unexplained symptoms (MUS) are often strained. Patients want explanations for their symptoms, and conflicts begin with the discrepancies in the interpretation of symptoms. Physicians often perceive patients with MUS as difficult, frustrating and demanding. Furthermore, patients are dissatisfied with the medical services they receive and feel misunderstood, disbelieved or rejected by their physicians. 
Claassen-van Dessel et $a l^{7}$ comment that $20-30 \%$ of active case loads in primary care are patients with MUPS. Some specialty clinics (eg, gynaecology, neurology) report rates as high as $66 \%$ of new visits. ${ }^{2}$

To date, a number of research-based treatment studies have been carried out, and although some have shown a small statistical improvement in patient outcomes, the clinical significance of these findings in community practice is unknown. Two recent Cochrane Reviews address this topic. One ${ }^{8}$ reported cognitive behavioural therapy as being more effective than usual care in reducing the severity of MUPS, but not more effective than enhanced care provided by the person's doctor. The other one, ${ }^{9}$ focused on pharmacologic interventions, concluded that the quality of current research was low and potential for bias high in many of the studies. This leaves the involved clinician with the quandary of what to do with this type of patient's troublesome and disabling symptoms.

Although a number of opinion-based management strategies have been published ${ }^{10-13}$ to guide physicians, there are no more comprehensive studies based on information obtained directly from practicing physicians.

In our qualitative study, we sample a group of clinical practitioners to examine and understand their experiences dealing with patients with MUPS within their clinical practices. Our study protocol simultaneously interviewed a series of patients who are/were experiencing MUPS, and this process added richness to the study as reciprocal insights were gained in the direct interviews and the data analysis. The fact that two of the researchers had also experienced MUPS added a depth of cross connection between caregiver and patient experiences and likely added a level of sophistication and in depth questioning that may have been otherwise lacking. The patient data have been published separately. $^{14}$ On the basis of the findings of four common themes, we propose an interim practical management guide (IPMG) for patients with MUPS which can be used to guide patient care while awaiting breakthroughs in better understanding the mechanisms behind this common problem and the development of more effective treatments. This interim guide also has the benefit of being easy to incorporate into practice.

A PubMed search (carried out on 12 February 2016) for articles published with an English abstract containing the terms 'medically unexplained physical symptoms' or 'medically unexplained symptoms' identified 173 references from 2000 to 2004, and 398 from 2011 to 2015. We theorise that the growth in interest relates to an increased recognition of the personal, social and economic costs associated with this condition for patients, physicians and society. ${ }^{15-17}$

\section{METHODS}

In a recent publication ${ }^{14}$ on patients with MUPS by our group, we reported in detail on the issues of methodological orientation and rationale, data collection, analysis and trustworthiness of our methods. As we used the same approaches in this study, the previous publication can be consulted for greater detail on the methodology.

\section{Approach}

This qualitative investigation was informed by a phenomenological research design, as we were interested in the essential qualities physicians experience in clinical encounters with patients with MUPS. We believed that privileging the primacy of individual experience was the most appropriate way to seek to understand an amorphous experience that by its very nature is fraught with uncertainty. That is, we saw the conceptualisation and treatment of MUPS as a phenomenon that can be only understood through the rich description of those engaged in the clinical encounter. One of the assumptions that framed the study was a recognition of our a priori experience in framing the issue. The synergistic convergence of these perspectives within the research team enhanced the conceptualisation of the phenomenon.

\section{Setting}

Two geographically separated teams participated in the research in parallel. A family doctor and a health services researcher composed team 1 at site A; while a neurologist, a political theorist and a communications graduate student made up team 2 at site $\mathrm{B}$. The research team felt that the disparate study sites were important to determine experiences that were intrinsic to the phenomenon as opposed to those that may be influenced by context. Both physicians had extensive professional experience dealing with patients with MUPS and both of the academic researchers had personal experience with MUPS, one in an acute situation and one chronically. The lived experiences of the team members brought credibility to the approach and interpretation and also gave rise to the need for additional levels of rigour to ensure that data collection and interpretation were open to new experiences. This was performed through 'memoing' the emerging relationship to the data by each researcher and regular check-in's to discuss interpretation. Memoing refers to the reflective note-taking the researcher does throughout the research process to enhance the credibility and trustworthiness of the data. $^{18} 19$ The research took place in two large urban centres in two Canadian provinces.

We obtained separate ethics approval from each academic institution. Prior to beginning the research interviews, we explained the study to the participating physicians and each physician signed a consent form to participate in the study.

\section{Recruitment}

As this was an exploratory study, we recruited physician purposefully. Our focus was on practitioners, known to 
the two physician members of the research team, who were likely to have had experience dealing with patients with MUPS. Potential participants were informed of the nature of the study and then were invited to participate. All invited participants agreed to participate in the interviews, and all were licensed practitioners in the province where the interviews took place.

\section{Data collection}

Interviews in both sites were performed by at least two of the team members and took place at a location convenient to the participants. In addition to questions around the demographics and background of participants, questions were asked to elicit the participants' cognitive framing of MUPS (eg, 'How do you manage a patient when you can't link their symptoms to any clear diagnosis?'); ethical and professional responsibilities; patient's rights within a clinical setting and decisions regarding communication. The interviews lasted between 45 and $90 \mathrm{~min}$ and were audio-recorded and transcribed for accuracy of interpretation.

\section{Analysis}

Members of the team at each centre reviewed their transcribed interviews independently using an open-coding approach and created a separate codebook from their data. Coding in qualitative research is an active inductive process that enables the researcher to identify, arrange and systematise the data, in our case the interview transcripts, in order to arrive at ideas, concepts and categories that explain the phenomenon. ${ }^{20-23}$ The approach by both teams followed standardised thematic coding and included starting with intense familiarity with the transcripts followed by the categorisation of data into tentative themes. Thematic coding, with its applicability across a range of theoretical and epistemological domains, allows 'theoretical freedom' while yielding a 'rich and detailed, yet complex, account of data'. ${ }^{24}$ The six-phased process of thematic coding proposed by Braun and Clark (data familiarisation, initial code generation, theme articulation, theme review, theme definition and narrative reporting) guided the coding. ${ }^{24}$

At this point team members reconciled the codebooks internally in each site. Subsequently, at a face-to-face meeting, team members created a single, revised codebook for the physician interviews. Once emerging themes were articulated, we each coded the entirety of the transcripts and then collaboratively created an explanatory narrative that adhered to the emerging data. The attention to a method of capturing each team members' perspective, without reducing meaning, led to a robust, interdisciplinary approach to the data, an essential attribute of the phenomenon being studied.

\section{RESULTS}

Twelve family physicians and 18 specialist physicians made up the study population. Box 1 details the variety of disciplines in the specialist physician sample. Most of the physicians came from urban centres.

On the basis of the described qualitative analysis, four key themes emerged from the physician interviews: (1) the challenge of diagnosis; (2) the challenge of management and treatment; (3) the importance of communication and (4) the importance of the therapeutic relationship. These themes were congruent with the priorities articulated by the patient arm of the study including the prioritisation of the therapeutic relationship in navigating the uncertainty that resulted from a lack of diagnosis, weighing the benefits of prolonged investigations, the importance of 'naming' and the eventual experience of acceptance or resignation. ${ }^{14}$ The multiple, overlapping key-stakeholder perspectives add robustness to understanding the multidimensional concept of MUPS. Each physician-generated theme will be commented on below.

\section{The challenge of diagnosis}

Patients with MUPS relate histories that rarely, if ever, fit the textbook descriptions of disease; there are no definite findings on physical examination and diagnostic testing is usually normal or unhelpful. At that juncture, the physician and patient have to decide what to do next: continue to pursue additional testing or opt for clinical follow-up. Physicians identified a number of potentially competing professional and personal factors affecting this decision.

Although many physicians mentioned their responsibility for resource allocation, its impact on decisionmaking varied. While many physicians feel a significant sense of responsibility to ensure appropriate allocation of health system resources, a minority does not. One physician stated categorically:

I don't feel at all that I'm the policeman for the system. B 28

Beyond considerations on the use of resources, the ethical principle of non-maleficence translated into the physicians' concern of the potential for iatrogenic physical harm being produced by the test procedures. According to one physician:

\section{Box 1 Specialties represented and numbers interviewed}

Emergency medicine, 1

Physical medicine and rehabilitation, 1

Respirology, 1

Palliative care, 1

Geriatrics, 2

Rheumatology, 2

Psychiatry, 3

Neurology, 3

General internal medicine, 4 
There are some patients who always want another test to the point that you begin to worry they're going to glow in the dark (referring to x-ray investigations). B7

Others recognised that iatrogenic harm in the form of harmful treatments could also result from false-positive test results. The following physician comment reflects that concern:

If the likelihood of your diagnosis is so low, it starts to creep up to our false positive rate in all our tests. It is dangerous. B6

The diagnostic period was a critical time for the physicians to demonstrate confidence in their capacity to navigate the challenges of patients with MUPS. Factors contributing to the physician's level of confidence included the depth of professional experience; confidence in their clinical skills; comfort in dealing with uncertainty and their understanding of the limits of medical knowledge. One physician described this as follows:

I think a lot of the patients tend to keep looking for an answer and they keep going doctor to doctor to doctor [...] And a lot of times you have to say, "Look. you know, we don't have the answer for everything. We don't have the tests for everything. And you know, common symptoms like fatigue and pain and things like that, we don't have any good tests" (for them implied). B8

Another physician commented:

I'm quite confident dealing with uncertainty. I don't feel the pressure. In fact the pressure to come up with a diagnosis is often an administrative one rather than a professional one. B12

\section{The challenge of management and treatment}

Once MUPS becomes the central diagnosis, the physician and patient need to achieve consensus with regard to management. In doing so, a number of issues emerge including the need to establish a shared management framework and outlining an agreed on mechanisms for follow-up. The importance of this is outlined in the following quote:

If we allow patients to kind of go to different places without sort of giving them sort of a framework to work in, I think that's where we really fail them. B17

In assessing reasonable options for treatment, physicians experience a tension between the art and the science of medicine. How these various factors come together provides the blueprint for the way an individual physician deals with uncertainty in conjunction with a particular patient.

We may not have an answer but we're both working towards the answer or we're at this point where we know we don't have an answer but we're willing to say, "Okay, fair enough, but I'll continue to try and help solve". But if that relationship changes then I'm not the person that's going to be helpful. I mean, it doesn't matter what the answer is after that, it doesn't matter because nobody is comfortable. A28

There is general agreement that the implementation of this care plan is best left to family physicians as the next two quotes suggest.

[...] what many of the primary care physicians are able to do is sort of recognize that-that this is a person that's going to be pushing for more investigations that aren't good for them. B11 (a specialist)

So I do think that family doctors look at these cases as an interesting challenge and we do not look at them as cases we can just wash our hands of if we can't explain [their symptoms]. A26 (a family doctor)

Requests for specialty consultations are usually aimed at ensuring no diagnosis is missed. However, in some cases, they are sought to provide support for the family physician's diagnosis and management plan. Specialist physician responses to the requests can be positive or negative. For example:

I have a great deal of respect for the physicians and the services that make referrals to me. They need to work with these patients. B10

or

For years I've said it's not my job to make the referring doctor feel bad. Every consult is valuable. Every single one of them is valuable. So it's not my job to say that was a dumb consult, that was totally inappropriate, no matter how inappropriate it might be. It's not my job to say that. B1

But

Some of them [the referring physicians] are afraid that if they explain what they think the diagnosis is, the patient won't be seen. BI

Levels of comfort with professional 'instinct', in the absence of clear evidence to aid diagnosis or treatment, varied among clinicians in this study. As one participant commented when discussing a MUPS patient:

How do you teach people, in an environment of evidence-based medicine, to live with the fact that either there is no evidence, or the evidence doesn't apply in your particular patient's case? A28

Another physician commented:

No, there's people [sic] that you see and you say, "I think there's something here. I'm not finding it." And, I mean in those situations, is usually where I try to get other 
people involved whose opinions I respect, and that would be where I'd probably go with those patients. B8

This is an especially challenging situation for physicians whose personal need for a diagnosis is strong. This concern is outlined in the following quote:

But sometimes the patients don't need a diagnosis and the doctor is the one that needs the diagnosis for their own mental comfort. B12

Even after a prolonged period of working with a patient, physicians expressed concerns about the possibility of missing a diagnosis for which a treatment is available. This anxiety sometimes created a reluctance to acknowledge the uncertainty with the patient, which in turn results in increased patient worry. One interviewee commented that doctor and patient needed to be aware of the vicious cycle that a desire for certainty can create.

[...] What we are doing is by not acknowledging uncertainty we are creating overly anxious people who want certainty in every encounter. A6

Having established the diagnosis of MUPS, doctors can offer patients a selection of either nonpharmacological or pharmacological interventions for symptom control. Non-pharmacological treatments usually involve the services of other allied health professionals (eg, physiotherapists, social workers, chiropractors, massage therapists, psychologists).

I like for people to think about alternative stuff. So, if there's lots of muscle spasms, chiropractic care, physiotherapy, warm baths, massage therapy, acupuncture can be useful. And for medications, I usually try to get them off of everything because they're usually on fifteen different meds. So I try to say over the next two years I'm going to try to get you off these things, because if they had cured you, you wouldn't be seeing me now. And um, some exercise, and consider your social circumstances and address those. B4

And

I think that some of these patients will benefit to a greater extent-once recognized and once serious things have been reasonably excluded-from non-medical health professionals. Physios who are interested, OTs, social workers, all that stuff which has become more difficult to access. B18

Pharmacological treatments are based on prescriptions and over the counter drugs. Owing to a lack of evidence that pharmacology may help, and in fact evidence that it may harm in the absence of a clear diagnosis, most participants in this study were cautious prescribing drugs with high risks of side effects such as narcotics. However, all physicians recognised the need for symptom control and used antidepressants, antiinflammatories or antineuropathic pain drugs on a trial basis. One participant spoke of his pre-emptive efforts to address any ongoing depression before he proposed diagnostic testing or other interventions:

If on day one when the patient came in and presented symptoms that didn't really fit with my academic model of disease I would tell them that on day one. I would say, 'You know you've presented me with some things that make me consider these things which are, you know, have to be investigated, and some of them are quite worrisome, but they're not that common, but we have to look at those. In the meantime ... there's something here that made me think of depression, and I think we should look at that today." A25

The importance of focusing on the social determinants of health such as diet and exercise and stress management is also important. As one physician noted:

I tend to focus on holistic stuff. A lot of people that I see they're overweight, they have terrible lifestyles, they smoke too much, drink too much, don't eat the right food, they get no exercise. ... I'll talk a lot about stress relief, because I think the chronic stress response plays a central role, not necessarily as the cause, but as part of the vicious cycle of ongoing illness and ongoing symptoms. B4

Several doctors employed ongoing assessment of the social and psychological well-being of patients with MUPS to act as benchmarks in determining the efficacy of any symptomatic treatments. Most of these measures of efficacy focused on improvements in quality of life. According to one clinician, what becomes most important is:

[.... How does this symptom interfere with your life, and what is it about it that distresses you the most, so that we actually end up with the result that the patient needs [are being met] at the end. A1

This tactic supported a 'harm reduction' approach; that is, having established the diagnosis of MUPS, physicians no longer aimed to cure, but rather encouraged the setting of realistic goals and the development of coping skills by the patient. When this takes place, a physician noted:

I do think that what happens in the end is that patients find ways to adapt and heal themselves. There is no external fix. A26

\section{The importance of communication}

Many of the challenges associated with caring for patients with MUPS focused on effective communication, particularly the conveyance of the physician's understanding of the reality of the clinical situation in a way that was persuasive for their patient.

[...] You hope to establish some sort of rapport with that person ... and it's not-nothing artificial-you actually 
do feel a sense of sort of empathy for the situation they are in. B11

Participants in this study felt that the diagnosis of MUPS must be discussed early and care taken to not present it as a diagnosis of exclusion. The recognition of the amount of uncertainty the diagnosis of MUPS creates for the patient must also be acknowledged. As one participant said:

To confront the patient with, "Well there is no neurological evidence that could possibly explain this," is not a helpful explanation [.... This is actually hurtful to some patients because they might assume that you're saying that they're lying, or that they're crazy, or that they have some other defect of their character that has led them to essentially waste your time. And I think those are messages that we don't want to encourage physicians to communicate. A 26

Clinicians approach the possibility of psychiatric referrals warily as their experience suggests patients with MUPS frequently hold the assumption that, when all their tests are negative, doctors assume that their symptoms are 'all in their heads'. Consequently, physicians may avoid mental health consultations, even when they think the consultations might be helpful. One physician noted the importance of such referrals:

But I think it's probably a good thing to refer them to a psychiatrist, because I can't imagine someone going through that-you know, when they have suffered so much and have so much changing their life, not going through some kind of reaction or change, you know. B15

Like most patients today, patients with MUPS go to the internet for medical information and then may request investigations or treatments they have discovered. While physicians generally valued the internet's contribution, especially in educating patients about specific, usually uncommon, diseases, it was less relevant for MUPS and created the need for physicians to serve as guides and interpreters of materials procured from websites.

[...] The Internet gives you massive information, but it doesn't tell you what's the most applicable information, there is a lot of junk there too. B18

In spite of how effective communication is, ultimately, there is a tacit awareness of the limits of medicine, the outer reaches of pathology that "medical science just hasn't figured out yet” B4.

\section{The importance of relationships}

Doctors characterised their relationship with patients with MUPS as complex because without solid 'evidence' to inform the way forward, clinical encounters become the focus of doctors' and patients' anxiety. The strength of the relationship between the two parties becomes key to any successful management of MUPS. Fostering empathy, compassion, trust and appropriate systematic availability encourages a substantive dimension to doctor-patient exchanges.

At its root, physicians interpreted empathy as an authentic acknowledgement of the reality of the patient experience and a belief in the impact of illness experience on overall quality of life. As one participant noted:

You mentioned empathy before, and it is really important with establishing the right sort of relationship to accompany a patient through that experience, and so when patients explain symptoms, I think that you have to have the trust that what they are experiencing is what they are experiencing. A4

Clinicians saw compassion as related to empathy, and patients with MUPS require compassion whether their symptoms are explainable or not. The substance and expression of compassion necessitate the building and maintenance of trust by the physician. The patient then trusts that the doctor believes that the patients' experiences are real, and thus they do not lose confidence in the capacity of their care provider. To be efficacious, all of this depends on the clinician's ability to spend adequate amounts of time with the patient to establish and sustain this therapeutic alliance. However, as described by one physician:

I think part of the problem is the system's so volume driven that being able to spend that time is a challenge. B17

Ultimately, participants in this study attested to the difficulty of treating patients with MUPS. They pose a challenge that, when managed badly, can have lasting consequences for the endurance and morale of the provider. As one physician candidly stated:

It's hard. One of those patients can burn you out for the rest of the day. $\mathrm{B} 2$

The following comment succinctly highlights the current dilemma physicians face when dealing with patients with MUPS.

[...] the profession needs to figure out first (what to do). That's the problem. Because we don't know what to do, You and I don't know what to do. We don't know what to tell these folks. B4

\section{DISCUSSION}

The emergent themes family physicians and specialty physicians identified in managing patients with persistent MUPS were similar and fell into four distinct groupings (see figure 1-emerging themes).

There was variability between individual responses in any one category. This variability was most noticeable when dealing with diagnosis and treatment issues and more precisely by comments about resource utilisation. 
Here, the remarks ranged from acknowledging the responsibility for taking costs into consideration when deciding what to do with an individual patient, to not considering costs at all. In contrast, statements emphasising the importance of communication and relationships were generally similar.

Even though cure for patients with MUPS remains elusive, the patients still need care. Currently, there is no guideline for the optimal way to provide this care based on data obtained from primary care and specialty practicing clinicians.

On the basis of our data, we propose the following guide for physicians involved with patients with MUPS (see box 2).

This interim guide is simple; does not require any specific technology; is not expensive for the patient; does not involve the outlay of dollars for the physician to implement and creates a guide to the provision of a care plan for the treating physician and the patient. It does mean there will be a need for more of the physician's time, which can be an issue.

In a recent paper, Howman et $a l^{25}$ report on general practice trainees' experience of managing patients with medically unexplained symptoms and the need to have better training to prepare them for clinical practice. The authors believe this interim guide would likely be as applicable to trainees as practicing physicians.

A number of factors suggest that our findings are valid and may be transferable to other contexts. First, in addition to the research which provides the data for this paper, in a similar study focusing on the experience of patients with MUPS, ${ }^{14}$ the patients highlighted needs similar to the recommendations for optimal care independently expressed by physicians. Second, despite the differences in populations, jurisdictions and locales, we found no thematic differences between the two research sites. Third, grounding the elusive concept of patients with MUPS in a large and varied group of physicians' lived experiences, we believe, contributes to the robustness of our data. Fourth, although there are no published studies similar to this study with which we could compare our results, our findings are generally in agreement with three opinion-based studies which have been published from England, ${ }^{10}$ the USA, ${ }^{11}$ and New Zealand ${ }^{12}$ and two studies based on surveys of attitudes of general practitioners to patients with MUPS. ${ }^{26}{ }^{27}$ Fifth, the diverse backgrounds of the researchers (a family physician, a neurologist, a communications graduate student, a health services researcher and a political theorist (the latter two who both had personal experience of MUPS)) and their involvement in all aspects of the study meant that the analysis of the data would have led to a much broader understanding of the phenomenon than could have been achieved by a team made up exclusively of physicians. Sixth, the very fact that two of the researchers had experienced MUPS and were part of the dyads (physician and researcher who had experienced MUPS) conducting patient and physician interviews likely led to a more nuanced and rigorous interview (and hence data set) than any previous study. The iterative development of insights resulting from each
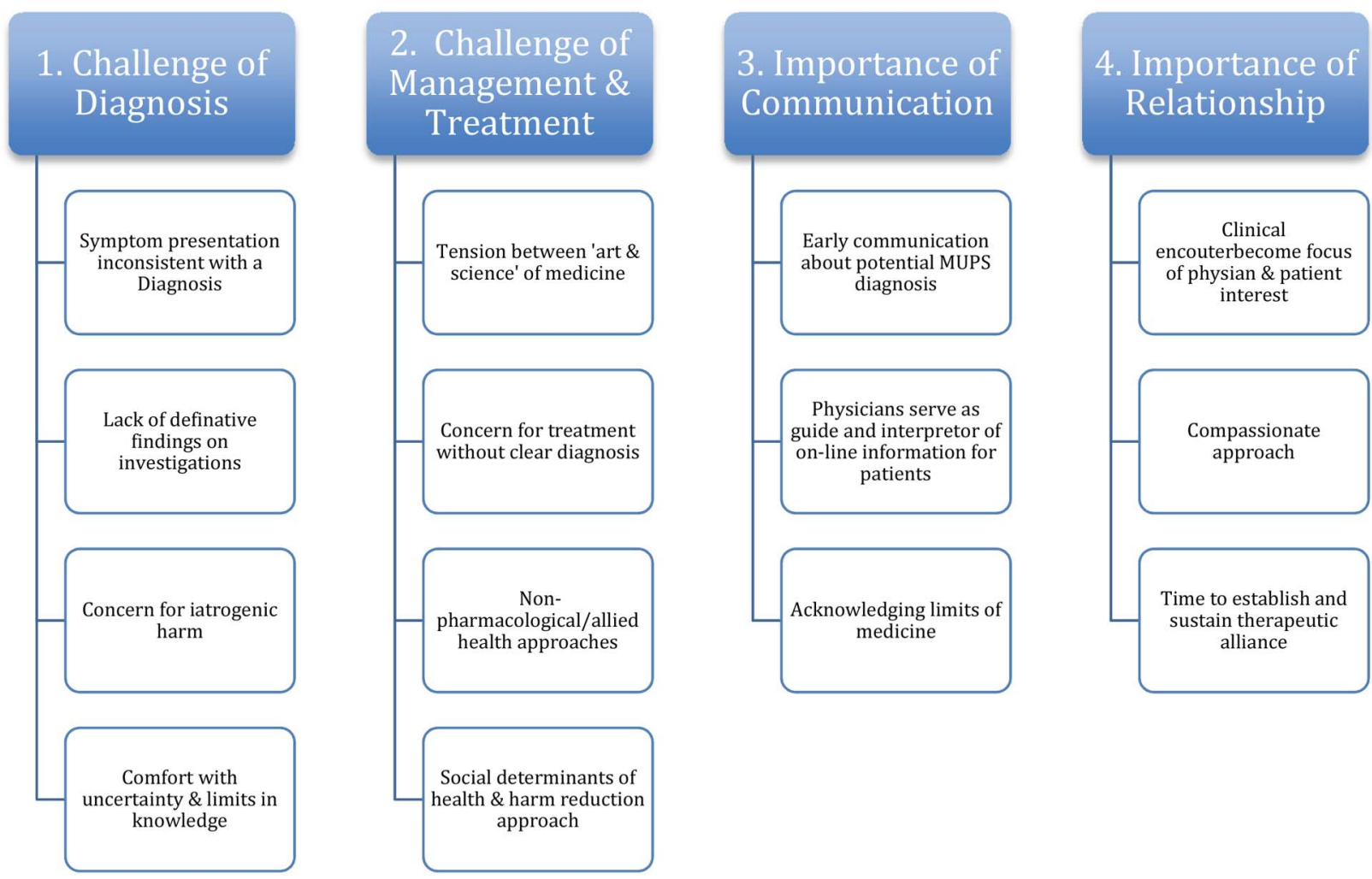

Figure 1 Emerging themes. 


\section{Box 2 Interim patient management guide}

1. Consider the diagnosis of MUPS early.

2. In consultation with the patient, limit the investigations to essentials.

3. Define the physician who will be responsible for provision of clinical follow-up.

4. Have that physician assure the patient of an ongoing commitment to care even if a diagnosis is not forthcoming.

5. Develop a care framework for the patient to follow, inclusive of supportive lifestyle approaches (ie, dietary changes, exercise, self-care).

6. Educate the patient about MUPS and the distinction between 'being resigned to their fate' and being committed to managing symptoms while enhancing the quality of their lives.

7. Avoid exposing the patient to potentially harmful treatments or invasive investigations.

8. Focus on the importance of communication and the relationship as key to the long-term management.

physician interview allowed researchers to individually and collectively refine their understanding of the MUPS experience while mutually retaining rigour in the methods. Finally, we also believe the method of data analysis contributed to the robustness of the findings. The methodology initially involved independent transcription review using an open-coding approach, followed by creation of codebooks. Subsequently, all members of the team, in a face-to-face meeting, developed a final codebook from which we reanalysed the transcriptions to develop an explanatory narrative for the data.

There are potential limitations to our study. First, since we limited the physician sample to family physicians and medical specialties that were less focused on technology, our conclusions may not be as applicable to surgical specialties and the technologically focused medical specialties such as cardiology and gastroenterology. Second, since adults were the focus of the study, we cannot comment on the relevance or applicability of our findings for paediatric patients with MUPS. Third, the physicians studied were primarily from urban locations. Fourth, the applicability of our findings to a nonpublically funded model of healthcare delivery may be different. Fifth, there has been no secondary validation of the proposed model.

Future prospective research needs to be performed to determine the utility of this model in a random selection of clinical practitioners from primary and specialty care who treat patients with MUPS. Similar studies could be performed to assess the utility of this model in general practice trainees. In addition, future studies that similarly explore patient and provider experience may provide further insights into the relationships that are central to successful caring for the patients.

\section{Author affiliations}

${ }^{1}$ Department of Clinical Neurosciences, Cumming School of Medicine, University of Calgary, Calgary, Alberta, Canada
${ }^{2}$ Department of Sociology, Faculty of Arts, University of Calgary, Calgary, Alberta, Canada

${ }^{3}$ Graduate Program in Communication and Media, Faculty of Arts, University of Calgary, Calgary, Alberta, Canada

${ }^{4}$ Department of Family Practice, Faculty of Medicine, University of British Columbia, Vancouver, British Columbia, Canada

Contributors JK and CA conceived and designed the study in collaboration with AKWB and RFW. JK and RFW conducted the interviews in centre $A$ and AKWB, CA and AW conducted the interviews in centre B. All authors had full access to the data in the study and can take responsibility for the integrity of the data and the accuracy of the data analysis.

Funding Canadian Institute of Health Research Catalyst Grant Ethics \# 245787. Competing interests None declared.

Ethics approval Ethics Committees at the University of British Columbia and the University of Calgary.

Provenance and peer review Not commissioned; externally peer reviewed.

Data sharing statement No additional data are available.

Open Access This is an Open Access article distributed in accordance with the Creative Commons Attribution Non Commercial (CC BY-NC 4.0) license, which permits others to distribute, remix, adapt, build upon this work noncommercially, and license their derivative works on different terms, provided the original work is properly cited and the use is non-commercial. See: http:// creativecommons.org/licenses/by-nc/4.0/

\section{REFERENCES}

1. Page LA, Wessely $S$. Medically unexplained symptoms: exacerbating factors in the doctor-patient encounter. J R Soc Med 2003;96:223-7.

2. Nimnuan $\mathrm{C}$, Hotopf M, Wessely S. Medically unexplained symptoms An epidemiological study in seven specialties. J Psychosom Res 2001;51:361-7.

3. Reid S, Wessely S, Crayford T, et al. Medically unexplained symptoms in frequent attenders of secondary health care: retrospective cohort study. BMJ 2001;322:767.

4. Burton C. Beyond somatization: a review of the understanding and treatment of medically unexplained symptoms (MUPS). $\mathrm{Br} J$ Gen Prac 2004;53:231-9.

5. Brown RJ. Introduction to the special issue on medically unexplained symptoms: background and future directions. Clin Psychol Rev 2007;27:769-80.

6. Aiarzaguena JM, Gaminde I, Clemente I, et al. Explaining medically unexplained symptoms: somatizing patients' responses in primary care. Patient Educ Couns 2013;93:63-72.

7. Claassen-van Dessel N, Velzeboer F, van der Wouden J, et al. Patients' perspectives on improvement of medically unexplained physical symptoms: a qualitative analysis. Ment Health Fam Med 2015;11:42-5.

8. van Dessel M, den Boeft M, van der Wouden JC, et al. Non-pharmacological interventions for somatoform disorders and medically unexplained physical symptoms (MUPS) in adults. Cochrane Database Syst Rev 2014;(11):CD011142.

9. Kleinstäuber M, Witthöft M, Steffanowski A, et al. Pharmacological interventions for somatoform disorders in adults. Cochrane Database Syst Rev 2014;(11):CD010628.

10. Mayou R. Medically unexplained physical symptoms. BMJ 1991;303:534-5.

11. Richardson RD, Engel CC. Evaluation and management of medically unexplained physical symptoms. Neurologist 2004;10:18-30.

12. Hatcher S, Arroll B. Assessment and management of medically unexplained symptoms. BMJ 2008;336:1124-8.

13. Kroenke K. A practical and evidence-based approach to common symptoms: a narrative review. Ann Intern Med 2014;161:579-86.

14. Kornelsen J, Atkins C, Brownell K, et al. The meaning of patient experiences of medically unexplained physical symptoms. Qual Health Res 2016;26:367-76.

15. Burton C, McGorm K, Richardson G, et al. Healthcare costs incurred by patients repeatedly referred to secondary medical care with medically unexplained symptoms: a cost of illness study. J Psychosom Res 2011;72:242-7.

16. Aamland A, Werner EL, Malterud K. Sickness absence, marginality, and medically unexplained physical symptoms: a focus-group study of patients' experiences. Scand J Prim Health Care 2013;31:95-100. 
17. Koola MM, Kuttichira P. Psychosocioeconomic study of medically unexplained physical symptoms. Indian J Psychol Med 2012;34: 159-63.

18. Groenewald T. Memos and memoing. In: Lisa M Given, ed. The SAGE encyclopedia of qualitative research methods. Thousand Oaks (CA): Sage, 2008:506

19. Corbin J, Strauss A. Basics of qualitative research (3rd ed.): techniques and procedures for developing grounded theory. Thousand Oaks (CA): Sage, 2008:117-42.

20. Benaquisto L. Codes and coding. In: Lisa M Given, ed. The Sage encyclopedia of qualitative research methods. Thousand Oaks (CA) Sage, 2008:86-8

21. Tracy SJ. Qualitative Research Methods : Collecting Evidence, Crafting Analysis, Communicating Impact (1). Chichester, West Sussex, UK: Wiley-Blackwell 2013: 189-202.

22. Krippendorff K. Content analysis: an introduction to its methodology 2nd edn. Thousand Oaks (CA): Sage, 2004:220. ISBN 9780761915454
23. Bourgeault I, Dingwall R, De Vries R. Why use qualitative methods to study health care organizations? Insights from multi-level case studies. In: Bourgeault I, Dingwall R, De Vries R, eds. The SAGE handbook of qualitative methods in health research. London: Sage, 2010:71-87.

24. Braun V, Clarke V. Using thematic analysis in psychology. Qual Res Psych 2006;3:77-101.

25. Howman M, Walters K, Rosenthal J, et al. "You kind of want to fix it Don't you?" Exploring general practice trainees' experiences of managing patients with medically unexplained symptoms. BMC Med Educ 2016;16:27.

26. Wileman L, May C, Chew-Graham CA. Medically unexplained symptoms and the problem of power in the primary care consultation: a qualitative study. Fam Pract 2002;19:178-82.

27. Reid S, Whooley D, Crawford T, et al. Medically unexplained symptoms--GPs' attitudes towards their cause and management Fam Pract 2001;18:519-23. 